\title{
Diseño social colaborativo para la creación de un programa de capacitación en Producción Audiovisual y Diseño Gráfico para jóvenes sordos
}

Mariana Lozada ${ }^{1}$ Mtr. Pontificia Universidad Católica del Ecuador

\author{
RECIBIDO: 22.02.2018 / ACEPTADO: 14.05.2018
}

\section{Resumen}

Este documento se presenta como una propuesta de reflexión sobre la aplicación del Diseño Participativo, tomando como referente la experiencia de uno de los proyectos de Servicio Comunitario a cargo de la Carrera de Diseño Gráfico de la Pontificia Universidad Católica del Ecuador, en el que se desarrolló una propuesta de enseñanza de Producción Audiovisual y Diseño Gráfico para jóvenes sordos de la Unidad Educativa Fe y Alegría Santo Domingo, en Ecuador.

Su objetivo está ligado a comprender la importancia del trabajo del diseño con una participación más cercana de y hacia los grupos beneficiarios, considerando la necesidad de establecer una propia metodología de diseño participativo para ser aplicada en proyectos posteriores.

Palabras clave: Diseño, social, educación, colaborativo, participativo.

\begin{abstract}
This document is presented as a reflection proposal on the application of Participatory Design taking as reference the experience of one of the Community Service projects in charge of the Graphic Design Career of the Pontificia Universidad Católica del Ecuador, in which work was carried out in develop a teaching proposal of Audiovisual Production and Graphic Design for deaf youth of the Educational Unit Fe y Alegría Santo Domingo, in Ecuador.
\end{abstract}

Its objective is linked to understanding the importance of design work with a closer participation of and towards the beneficiary groups, considering the need to establish a methodology of participatory design to be applied in subsequent projects.

Keywords: Design, social, education, collaborative, participatory.

\footnotetext{
1 Docente Universidad Católica desde 2015, Consultora y capacitadora de Gestión de marca estratégica en Designers with heart, Experta de Comunicación en SERCOP, Gerente del Proyecto SECOM Itinerante en la Secretaría Nacional de Comunicación; Jefe de Comunicación, Responsable de Relaciones Interinstitucionales, Responsable de Planificación, en Fundación Museos de la Ciudad; Coordinadora de Facultad de Diseño, Coordinadora de responsabilidad social y vínculo con la colectividad, docente de diseño en Universidad Tecnológica Israel. mablozada@gmail.com
} 


\section{Introducción}

Como precedente, es importante indicar que en los últimos años, tanto en el ámbito profesional como académico, se ha empezado a hablar cada vez más sobre el diseño enfocado en las personas; pero, ¿Acaso el diseño no se ha centrado siempre en las personas? Muchos utilizan este término para mencionar un modelo de diseño socialmente responsable, cuyo principal objetivo es la satisfacción de las necesidades humanas y el aporte en la mejora de su calidad de vida, poniendo especial cuidado en los sectores de atención prioritaria.

Para este texto, la descripción mencionada se tomará como una responsabilidad implícita en el ejercicio profesional de todo diseñador y como la base para la ejecución de una propuesta de diseño social específica. Esto se fundamentaría en el análisis de las responsabilidades y el ejercicio ético del diseño que propone Findeli en su texto Rethinking Design Education for the 21st Century: Theoretical, Methodological, and Ethical Discussion, donde indica: "El diseño no solo podría contribuir a un mundo natural sostenible, sino que también adopte como propósito algo como: Una humanidad equilibrada en un mundo equilibrado..." (Findeli, 2001)

Para empezar, es importante mencionar que a través de las metodologías de diseño de diversos autores, existen varias formas de trabajar en el diseño, una de ellas es el trabajo participativo, donde la interacción con las comunidades es una de los factores principales y donde el análisis interdisciplinar, así como el contacto directo con los espacios, contextos e intervención directa de los actores involucrados en el proceso, propone soluciones acorde al ámbito específico de aplicación, enfocadas en las necesidades propias de las comunidades beneficiarias.

En el caso específico del proyecto de servicio comunitario "Laboratorio audiovisual Fe y Alegría" ejecutado entre la Pontificia Universidad Católica del Ecuador (PUCE) y la Asociación Fe y Alegría del Ecuador, siendo el primero que se ejecuta teniendo como cabeza a la Carrera de Diseño, se evidencia con toda claridad el proceso de trabajo mencionado, pero se experimenta al mismo tiempo una necesidad latente de establecer una metodología propia de trabajo colaborativo que se ajuste a los rasgos característicos de los proyectos comunitarios de la Universidad.

\section{Antecedentes}

La redacción de este documento parte de una reflexión acerca del diseño social en el marco del Diplomado en Diseño e Innovación Social de la Universidad Rafael Landívar de Guatemala, donde se presentaron varios casos de estudio vinculados a nuevas maneras de hacer diseño desde una visión más colectiva, meditando sobre cómo la sociedad lo piensa tanto desde una visión global como desde la concepción procedimental de los propios diseñadores, analizando hacia donde apunta el de estos profesionales, y pensando en la necesidad de que la sociedad deje totalmente de lado la percepción del diseño como un tipo de práctica artística que permite realizar productos "bonitos", sino que la identifique como una disciplina de impacto comunicacional, operacional y de contribución al bienestar social.

También es importante hacer que los diseñadores sepan que se enfrentan a una nueva manera de ver su profesión, ya no solo desde el ámbito mercadológico y comunicacional, sino también a través de propuestas 
que se vinculan con un sin número de áreas del conocimiento que hasta el momento se veían muy lejanas, y que enriquecen su percepción del entorno y mejoran su capacidad de "diseñar para la gente".

Ahora, para la descripción de este proyecto comunitario, es importante mencionar que la investigación parte de un cambio de paradigma nacional en el que el Gobierno Nacional del Ecuador establece nuevas propuestas inclusivas de educación en beneficio de las personas con discapacidad, poniendo especial cuidado en las acciones propuestas desde los entornos universitarios, es así que, a través de la participación de varios delegados de la academia, se genera un documento denominado "Construyendo igualdad en la educación superior", el que propone fundamentos y lineamientos para transversalizar los ejes de igualdad y ambiente en el entorno de las universidades ecuatorianas y su gestión cotidiana.

A partir de esto es importante entender cuál es el eje conceptual institucional que da paso a este tipo de producciones y como el diseño se inserta como un factor clave de acción. En el caso puntual del proyecto "Laboratorio audiovisual Fe y Alegría", parte de una iniciativa conjunta entre dos instituciones jesuitas brevemente presentadas a continuación.

Fe y Alegría Ecuador es una institución sin fines de lucro que promueve la educación popular y de promoción social, para que hombres y mujeres de sectores desatendidos del país aprovechen sus potencialidades para mejorar o transformar la realidad que los rodea.

Por su parte, la PUCE según su departamento de Vínculo con la Colectividad

"es una comunidad académica que, de modo riguroso y crítico, contribuye a la tutela y desarrollo de la dignidad humana y de la herencia cultural mediante la investigación, la docencia, la vinculación con la colectividad y la formación complementaria a través de los diversos servicios ofrecidos a las comunidades locales e internacionales.

El espíritu que impulsa la Vinculación con la Colectividad en la PUCE es la búsqueda de la promoción de la justicia, la solidaridad y la equidad social para la transformación de la sociedad a través de los criterios de Responsabilidad Social Universitaria definidos por la AUSJAL, red a la que pertenece la Universidad."

El proceso inicia cuando Fe y Alegría identifica varios inconvenientes laborales que enfrentan sus egresados sordos, puesto que, a pesar de su formación, se insertan en un entorno social poco inclusivo que les genera oportunidades laborales donde la aplicación de sus conocimientos académicos se reduce considerablemente, hasta el punto de tener apenas acceso a labores vinculadas a la limpieza, artesanía, agricultura, que aun cuando no son en ningún sentido denigrantes, limitan su capacidad de aplicar sus potencialidades profesionales.

Con este antecedente, Fe y Alegría, preocupada por la situación laboral de los estudiantes sordos que egresan concretamente de una de sus unidades educativas de educación media ubicada en ciudad de Santo Domingo, perteneciente a la provincia costera de Santo Domingo de los Tsáchilas, decide acercarse a la PUCE con el fin de fortalecer su gestión inclusiva dentro del país.

Es así que ambas entidades firman un convenio interinstitucional que les permite trabajar de forma conjunta para implementar el proyecto "Laboratorio audiovisual Fe y Alegría" y contribuir con este al cumplimiento de 
varios de los objetivos del Plan Nacional del Buen Vivir ligados al tema de la inclusividad, la educación y la mejora de la calidad de vida de los ecuatorianos, en este caso a través de la dotación de conocimientos y herramientas de diseño y producción audiovisual para que los egresados sordos de la Unidad Educativa Fe y Alegría Santo Domingo puedan insertarse en el ámbito profesional en mejores condiciones y con mayores oportunidades laborales.

\section{Aporte universitario}

El proyecto "Laboratorio audiovisual Fe y Alegría" se concibe como un proceso de tres fases vinculadas a la educación, retroalimentación y auto sustentabilidad de los estudiantes sordos que egresan de la Unidad Educativa Fe y Alegría Santo Domingo. Las limitaciones que se identificaron previo al inicio del proyecto son:

a.- Los profesores de la Unidad Educativa Fe y Alegría Santo Domingo no poseen el conocimiento técnico que determine las acciones que se deben realizar para adecuar el espacio físico donde se prevé que funcione el Laboratorio Audiovisual, tampoco poseen un equipo asesor para la compra de equipos audiovisuales que se requieren para la implementación del laboratorio.

b.- Los profesores de Fe y Alegría que forman parte de este proyecto poseen formación en educación general (conocimientos técnicos de informática y paquete Office), más no de asignaturas vinculadas al Diseño Gráfico o Producción Audiovisual.

c.- Fe y Alegría no posee el material de apoyo necesario para impartir las clases referentes al Diseño Gráfico y Producción Audiovisual.

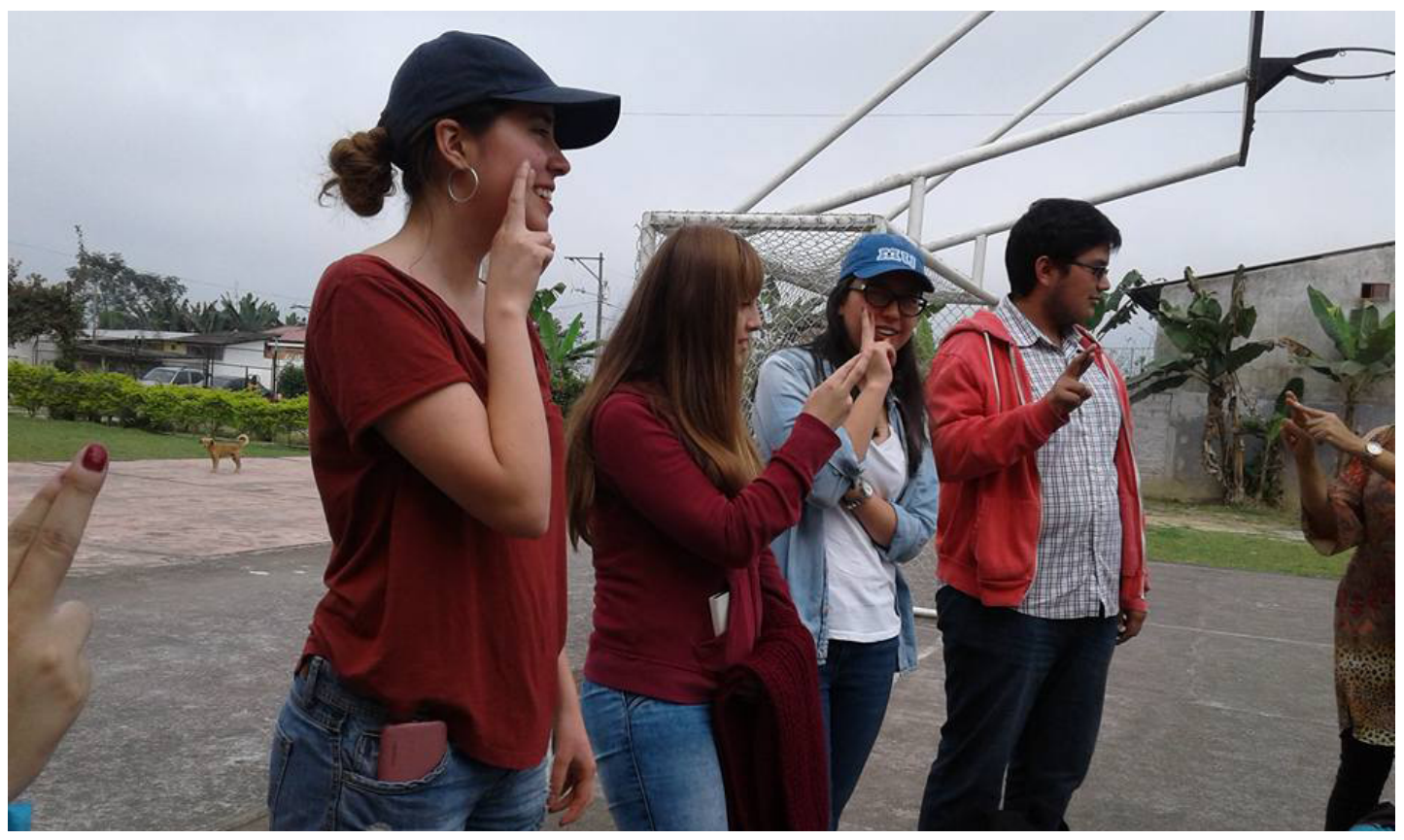

Fig. 1. Primer acercamiento a la comunidad. 
Bajo este contexto, los representantes de Fe y Alegría solicitan el apoyo de la PUCE con el fin de brindar asesoría y capacitación respecto a la implementación del laboratorio, para ello se plantean los siguientes objetivos:

\section{Objetivo general}

Contribuir a través de la capacitación y asesoría técnica en la consolidación de espacios inclusivos de formación y emprendimiento para personas con discapacidad auditiva dentro del Proyecto Laboratorio Audiovisual de la Asociación Fe y Alegría.

\section{Objetivos específicos}

- Brindar asesoría técnica respecto a la adecuación de espacios físicos donde funcionará el Laboratorio Audiovisual, así como de los equipos técnicos y software necesarios para su utilización.

- Capacitar a 10 docentes y a 10 ex estudiantes de Fe y Alegría en asignaturas vinculadas al Diseño Gráfico y Producción Audiovisual.

- Desarrollar material de apoyo visual para la emisión de clases por parte de los profesores capacitados.

- Acompañar en el proceso de retroalimentación y consolidación del emprendimiento.

\section{Actores directos}

Fe y Alegría: directivos nacionales, coordinadores de proyecto, directivos de la Unidad Educativa, profesores, estudiantes, ex estudiantes y padres de familia.

PUCE: directivos, coordinadores de vínculo con la colectividad, profesores y estudiantes de las Carreras inmersas (Arquitectura, Diseño Gráfico y Comunicación).

\section{Actores indirectos}

Gobierno Nacional: Ministerio de Educación; Secretaría Nacional de Educación Superior, Ciencia, Tecnología e Innovación (SENESCYT); Consejo de Evaluación, Acreditación y Aseguramiento de la calidad de la Educación Superior (CEAACES); Consejo de Educación Superior (CES); Instituto Nacional de Audición y Lenguaje (INAL); Consejo Nacional para la Igualdad de Discapacidades (CONADIS).

Municipalidad: Autoridades y población de Santo Domingo de los Tsáchilas.

Otros actores: otras universidades, resto de la PUCE, resto de Fe y Alegría Ecuador, otras instituciones ligadas a la discapacidad, población de Quito. 


\section{Modalidad}

Posterior a la firma del convenio interinstitucional, los coordinadores de cada institución buscaron información y definieron la viabilidad del proyecto. Posterior a ello presentaron la propuesta de trabajo, la que después de ser aprobada, se socializaron con los actores involucrados en cada Institución.

Respecto a la ejecución del proyecto se plantearon tres fases que se detallan a continuación:

\section{Fase 1}

Diseño e implementación de un espacio físico donde funcione el Laboratorio audiovisual, en este caso el planteamiento arquitectónico fue responsabilidad de los estudiantes de la PUCE Quito.

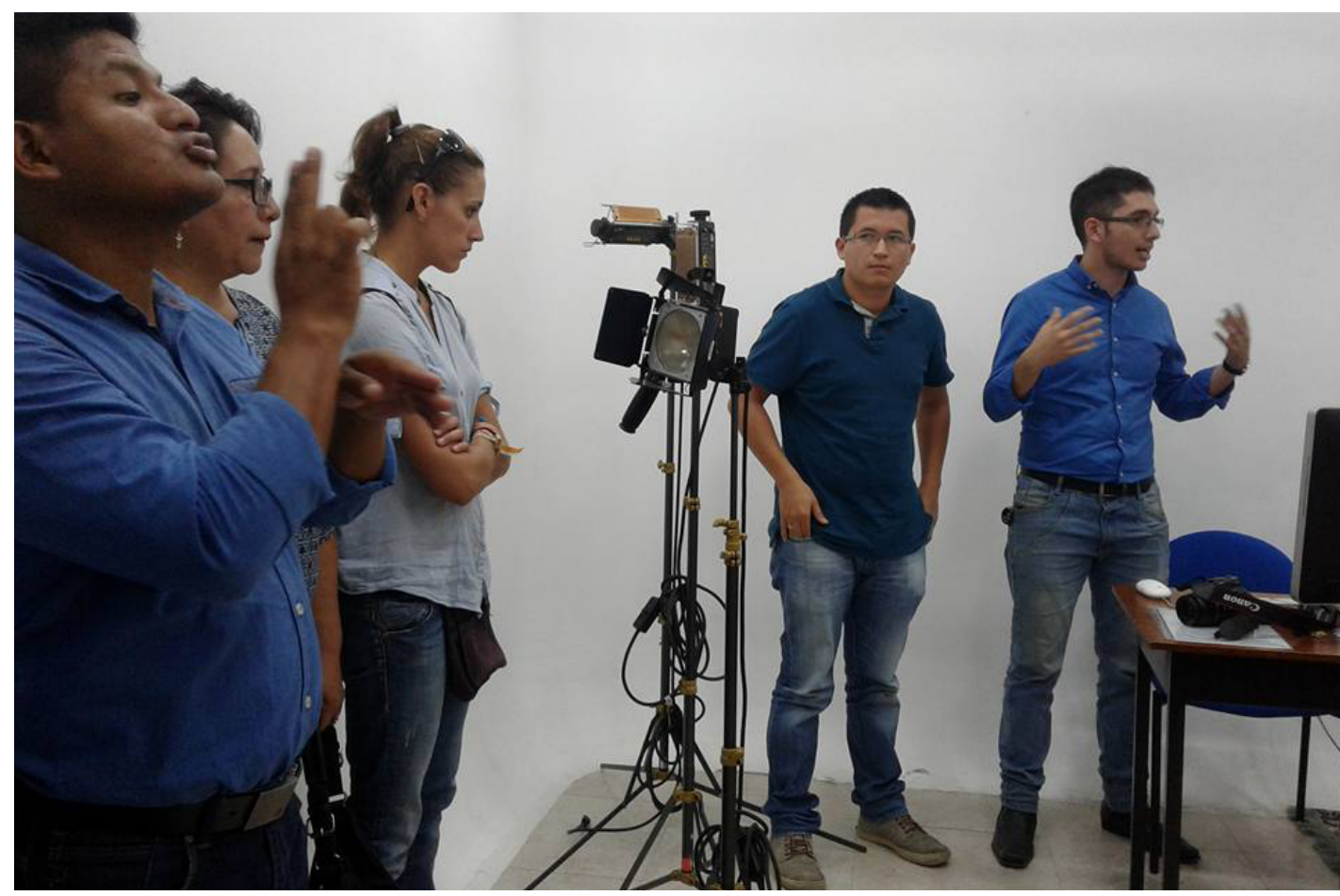

Fig. 2. Visita a las áreas de trabajo de la PUCE, Santo Domingo.

Visitas de observación de los estudiantes de Fe y Alegría a las instalaciones de la PUCE Santo Domingo, con el fin de fortalecer los vínculos entre ambas instituciones e identificar aspectos que mejoren las condiciones de interacción entre los actores del proyecto para tener mayor certeza de las acciones que se podrían aplicar.

Desarrollo de material de estudio sobre diseño y producción audiovisual inserto en la plataforma digital de Fe y Alegría, a través del cual los estudiantes sordos de Fe y Alegría con el apoyo de tutores especializados, recibirán instrucción on-line, para posteriormente complementar conocimientos a través de clases presenciales, es decir las capacitaciones se ejecutarán en modalidad semipresencial. Esta fase fue ejecutada por los estudiantes de Diseño Gráfico y Comunicación de PUCE Quito. 


\section{Fase 2}

Jornadas de capacitación semipresencial a los estudiantes sordos, mediante el soporte y asesoría conjunta de los estudiantes de PUCE Quito y Santo Domingo.

Espacios de acompañamiento a la réplica de los procesos de enseñanza - aprendizaje hacia los nuevos estudiantes de Fe y Alegría, con el apoyo de profesores y estudiantes de PUCE Santo Domingo.

\section{Fase 3}

Implementación de una cartera de servicios en Diseño y Producción Audiovisual dentro del Laboratorio audiovisual, la misma que será ejecutada por los estudiantes sordos que asistieron a las capacitaciones dictadas por los estudiantes de la PUCE. Esta fase se ejecuta también con el soporte de estudiantes de PUCE Santo Domingo, quienes forman parte del equipo bajo la figura de prácticas pre profesionales y cumplen la función de asesorar a los chicos sordos.

\section{Ejecución}

Para la ejecución de la primera fase se buscó que previo a la implementación, cada actor genere aportes de acuerdo a su expertís en el tema y se desarrolló proceso de observación participante y no participante con el fin de entender el contexto de aplicación y los rituales de las personas sordas, se ajustó el proyecto de acuerdo a la información encontrada y se estableció la metodología, técnicas y herramientas de aplicación de la propuesta.

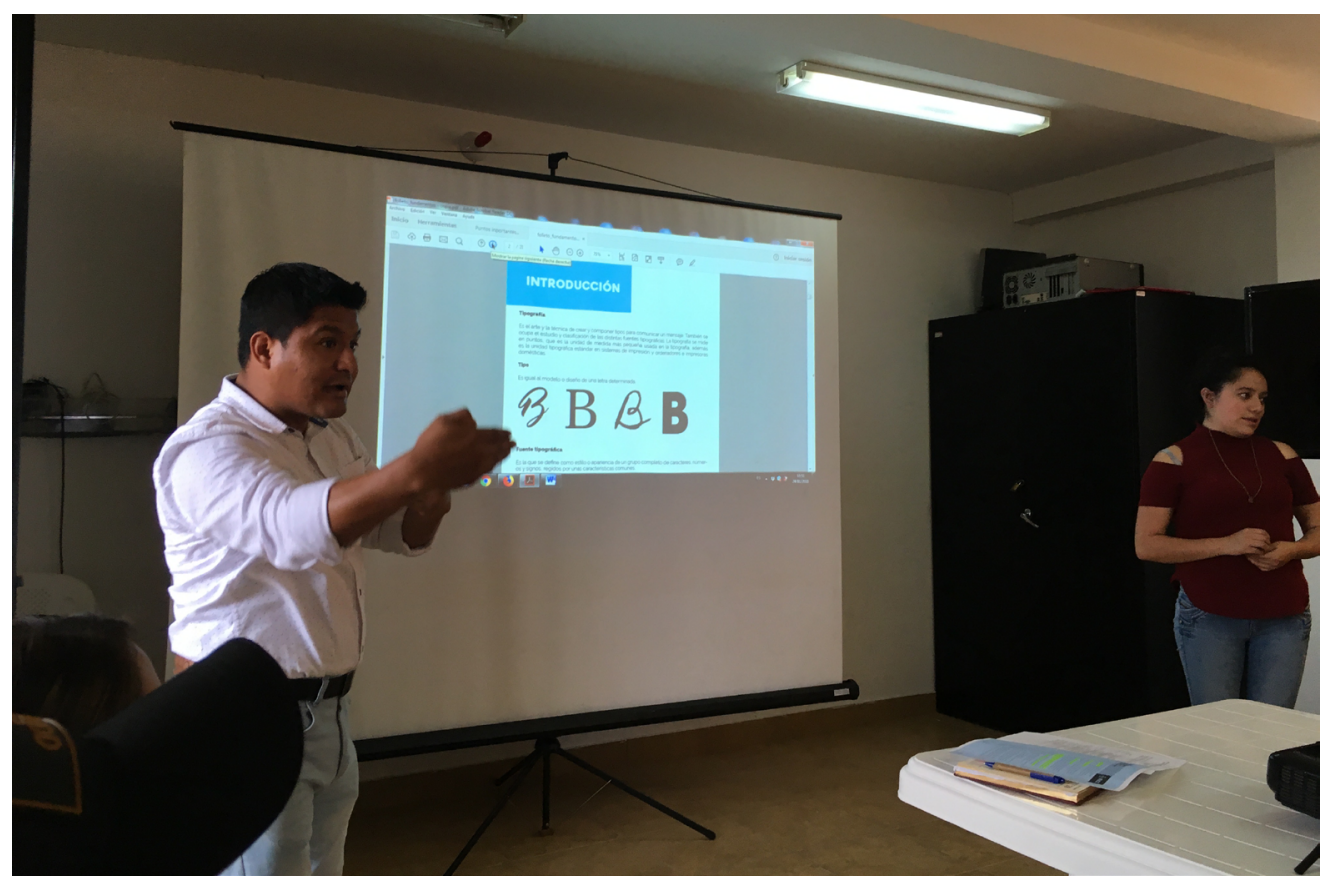

Fig. 3. Entrega del material educativo.

Durante el primer semestre del proyecto se trabajó entre los coordinadores de cada Institución, docentes y estudiantes de la PUCE y docentes de la Unidad educativa de Fe y Alegría en la preparación del material de estudio con el que se alimentó la plataforma de aprendizaje de herramientas de diseño y producción 
audiovisual y se presentó los contenidos a las personas encargadas de la parte educativa en Fe y Alegría, así como a los estudiantes sordos interesados en ser parte de este plan piloto.

Es importante recalcar que el aporte pedagógico y especializado en temas de sordera por parte de profesionales tanto de Fe y Alegría como de la PUCE, fueron un puntal para mejorar el material inicialmente propuesto.

Cabe recalcar que el proyecto no está vinculado a la generación de diseñadores express, sino más bien se enfoca en dotar a las personas sordas de conocimientos base de diseño que les permitan tener una habilidad adicional a su formación secundaria y les abra las puertas a nuevas oportunidades de trabajo como posibles operarios del diseño; $y$, si las posibilidades lo facilitan, darles la oportunidad para que en un futuro puedan inclinarse por el estudio formal de una carrera en diseño.

Al momento el material fue presentado a los especialistas en educación inclusiva, autoridades de Fe y Alegría, docentes y estudiantes sordos interesados en el proyecto, obteniendo una gran aceptación por los mismos y entendiendo que la generación de material educativo dependerá de varios factores característicos del público al cual se lo esté enfocando; en el caso de los jóvenes sordos, es fundamental entender su realidad y adentrarse en su espacio, en su día a día, en sus conflictos y limitaciones para identificarlos y generar propuestas más efectivas.

Se espera que en los próximos meses se pueda iniciar la fase dos, donde seguramente se evidenciarán aspectos que permitirán modificar la propuesta y ajustarla a las necesidades del público objetivo.

\section{Análisis del contexto. Sobre la Innovación y el Diseño Social}

La importancia de este tipo de proyectos, es que forma parte de un nuevo modelo de cambio social y cultural, ya que interfiere directamente en los comportamientos y las relaciones humanas.

Es importante que toda iniciativa de diseño, no solo considere los entornos políticos, sociales, legales, de acción colectiva y de política pública, sino que le de igual importancia a aquellos aspectos que le permitan entender al ser humano como un sujeto con características propias y modelos de vida específicos.

Bajo este contexto, el trabajo con los jóvenes sordos de Fe y Alegría parte de una concepción del diseño como una propuesta aplicable para todo tipo de personas, tomado como eje El libro blanco para todos en las universidades, que menciona:

"Diseño para Todos (Design for All), que centra su actividad en la búsqueda de soluciones de diseño para que todas las personas, independientemente de la edad, el género, las capacidades físicas, psíquicas y sensoriales o la cultura, puedan utilizar los espacios, productos y servicios de su entorno y, al mismo tiempo, participar en la construcción de éste.

Por tanto, el Diseño para Todos es una herramienta útil para hacer entornos accesibles que permitan el desarrollo individual de todas las personas." Aragall F. et al. (2006, p.28). 
Findeli también se refiere a que el diseñador posee una tarea de comprender la dinámica de los sistemas en los que interactúa, hace hincapié en que no puede actuar solo o contra el sistema sino que es necesario que se integre al sistema para motivar su funcionamiento. (Findeli, 2001).

Además, es sustancial considerar que este tipo de propuestas implica una participación absoluta de todos los actores, para lo cual se debe procurar un entorno accesible, respetuoso, seguro, saludable, funcional, comprensible y estético, como lo indica el texto mencionado.

Esto se complementa también con lo que menciona la Segunda Edición del Kit de Herramientas de Diseño Centrado en el Usuario:

"ayudará a escuchar de un modo diferente a los miembros de la comunidad cuando expresen sus necesidades, a crear soluciones innovadoras que den respuesta a esas necesidades y a poner en práctica soluciones, teniendo en cuenta la sustentabilidad financiera." (IDEO, s.f., p. 3)

Con estas premisas para inicial el proyecto, se analiza también el diseño educativo, como una de las herramientas de alto impacto en cualquiera de las etapas de adquisición de conocimientos del ser humano, sea este formal o no formal, que trasciende el proceso de enseñanza - aprendizaje, como lo menciona Frascara, al afirmar que la educación "va más allá de la adquisición de habilidades y conocimientos existentes, sino que se dirige al desarrollo de las personas, proceso en el que la participación activa del usuario del diseño es indispensable" (2012, p.139).

En el caso específico de este proyecto, los textos citados sustentan la necesidad de establecer una modalidad coparticipativa que permita insertar una nueva alternativa de diseño educativo en la que se considere la interpretación personal del estudiante sobre el mundo que lo rodea y su relación con el evento estudiado. Esa visión hace que el proceso de construcción de conocimiento ocurra de una manera más cercana al individuo y este vea el resultado como una posibilidad de satisfacer sus necesidades.

Al ser un proceso participativo y de aplicación, es más fácil generar iniciativas de diseño variadas, innovadoras y de mayor alcance. Bajo este contexto, las herramientas visuales en los roles pedagógicos son de suma importancia, ya que el impacto de las propuestas visuales incrementa en gran manera la facilidad de aprendizaje de los estudiantes.

Para este proyecto de gestión comunitaria se utilizó la metodología proyectual, considerando que la investigación y la propuesta final se inserta en etapas de trabajo donde se busca alcanzar de forma gradual y recursiva los objetivos planteados en cada momento.

El modelo de investigación del "Laboratorio audiovisual Fe y Alegría" se vinculó a un proceso de identificación de la problemática, justificación y objetivos, recopilación y análisis de información, desarrollo del proyecto a través de una metodología de diseño específica, evaluación de resultados, validación. Durante todo el proyecto se motivó la reflexión teórica individual y colectiva, promoviendo el pensamiento crítico y el compromiso de todas las partes involucradas.

Este proceso investigativo evidencia los grandes compromisos del diseñador desde los ámbitos profesionales, 
éticos, sociales y culturales y le permite a la Universidad insertarse en un campo de estudio que hasta el momento ha sido muy poco explorado desde esta disciplina.

Con lo mencionado se propone dar cumplimiento al objetivo del Diseño para todos, que describe "que todas y cada una de las personas tengan las mismas oportunidades a la hora de escoger un trabajo, una actividad, una residencia o un estilo de vida." Aragall F. et al. (2006, p.43).

Pero aun cuando se logró trabajar con una metodología educativa y una buena estructura de planificación participativa, no fue fácil identificar una metodología de diseño participativo que se ajuste a los procesos de Servicio Comunitario de la PUCE.

A lo largo de este proyecto, se investigaron diversos sistemas de trabajo colaborativo como el propuesto por las docentes Ana Cielo Quiñones y Gloria Barrero, de la Universidad Javeriana de Bogotá; la adaptación del modelo Colores por la vida de la Universidad Javeriana de Cali, el ajuste de metodologías tradicionales de diseño o modelos ya preestablecidos como la consideración de los siete principios del diseño universal, pero es importante entender que cada espacio posee su lógica y requiere de tiempo y ensayo para conseguir el establecimiento de una metodología propia.

En el caso de la Carrera de Diseño, esta experiencia ha sido un puntal para que se abra las puertas a nuevos proyectos que partan desde el Diseño, ya no como un complemento a otras áreas del conocimiento, o como una manera de hacerla atractiva, sino como un eje trascendental para dotar a la sociedad de soluciones efectivas y sostenibles.

El propósito para el 2018 es gestionar propuestas de Diseño de Servicio Comunitario que se fundamenten en una metodología de trabajo colaborativo propio que sirva de referente para otras universidades del país.

\section{Conclusiones}

En su ejercicio diario, el diseñador tiene la posibilidad de escoger varios modelos de trabajo, pero es fundamental analizar bien el modelo seleccionado y saber que cualquiera que se use es susceptible a cambios o adaptaciones sustentadas según el espacio en el que se trabaje.

El diseño no funciona solo, es utópico pensar que tiene la capacidad de dar soluciones completas. Es necesario aprender a trabajar en equipo, buscar soporte en las áreas de la interdisciplina que complementen y fortalezcan el trabajo, así como tener los sentidos prestos para captar la sensibilidad de los entornos de intervención.

Es importante que el diseñador mismo comprenda la trascendencia de su labor y como esta ha ido cambiando, de tal manera que pueda interiorizar su importancia y logre que la sociedad también considere a esta disciplina como una herramienta fundamental de transformación social. 


\section{Bibliografía}

ARAGALL F. ,BENENTI, B., FERRER. J., FORT, J.M., HERNÁNDEZ, J., MERODIO, J.R., MONGUET, J.M., POL, E. Libro Blanco del Diseño para Todos en la Universidad (1ª ed.). España: Fundación ONCE e Instituto de Mayores y Servicios Sociales (IMSERSO), 2016.

BARRERA G. , QUIÑONES A. (2009) Diseño socialmente responsable: ideología y participación. Bogotá, D.C.: Pontificia Universidad Javeriana, Facultad de Arquitectura y Diseño.

CHRISTOPHERSEN J. . Universal Design: 17 Ways of Thinking and Teaching. Husbanken, 2012. FINDELI A. "Rethinking Design Education for the 21st Century: Theoretical, Methodological, and Ethical Discussion" en Design Issues, Vol. 17, № 12001, pp. 5-17.

FRASCARA J. El diseño de comunicación. (1ํe ed.), Buenos Aires: Infinito 2012.

FRASCARA J. Diseño gráfico para la gente. Comunicaciones de masa y cambio social. (1를. ed.), Buenos Aires: Infinito 1997.

HERDOÍZA M. Construyendo Igualdad en la Educación Superior. (1ae ed.), Quito: Senescyt/Unesco 2015. IDEO. (s.f.) Diseño Centrado en las personas (2 ${ }^{\mathrm{a}}$ ed.), S.I.: S.ed.

PAPANEK V. Diseñar para el mundo real. ( $2^{\mathrm{a}}$ ed.), en Barcelona: Pol-len edicions 2014.

PELTA R. Diseñar con la gente. ELISAVA TdD, № 24, 2007, pp. 27-34.

PONTIFICIA UNIVERSIDAD CATÓLICA DEL ECUADOR (PUCE). Borrador instructivo para regular las prácticas pre profesionales y el servicio a la comunidad de la PUCE, 2017.

MOULAERT F. Towards Alternativ Model(s)of Local Innovation enUrban Studies, vol. 42, no 11, 2005, pp. 1969-1990.

RAMÍREZ R. Guía de buenas prácticas de diseño: herramientas para la gestión del diseño y desarrollo de productos. (1를. ed.), San Martín: Inst. Nacional de Tecnología Industrial - INTI 2012.

SÁNCHEZ A. Diseño colaborativo: abriendo el proceso de diseño. Revista +MAS D, v 2013, pp. 1 -11.

SHEDROFF N. Creación de emociones, significados y experiencias en Revista Faz, oㅡㄹ, 2008, pp. 8-97.

\section{Fuentes de las imágenes}

Fig. 1. Primer acercamiento a la comunidad. Fotografía del Equipo de Servicio Comunitario PUCE Discapacidades.

Fig. 2. Visita a las áreas de trabajo PUCE Sto. Domingo. Fotografía del Equipo de Servicio Comunitario PUCE Discapacidades.

Fig. 3. Entrega del material educativo. Fotografía del Equipo de Servicio Comunitario PUCE Discapacidades. 